\title{
Macroscopic acousto-mechanical analogy of a microbubble
}

\author{
Jennifer Chaline, ${ }^{1, a)}$ Noé Jiménez, ${ }^{2}$ Ahmed Mehrem, ${ }^{2}$ Ayache Bouakaz, ${ }^{1}$ \\ Serge Dos Santos, ${ }^{3}$ and Víctor J. Sánchez-Morcillo ${ }^{2}$ \\ ${ }^{1}$ UMR Inserm U930, Université François-Rabelais, 10 boulevard Tonnellé, 37032 Tours, France \\ ${ }^{2}$ Instituto de Investigación para la Gestión Integrada de Zonas Costeras, Universitat Politècnica de València, \\ Paranimf 1, 46730 Grao de Gandia, Spain \\ ${ }^{3}$ INSA Centre Val de Loire, UMR Inserm U930, Université François-Rabelais, 3 rue de la Chocolaterie CS \\ 23410, F-41034 Blois, France
}

(Received 12 August 2015; revised 29 October 2015; accepted 16 November 2015; published online 16 December 2015)

\begin{abstract}
Microbubbles, either in the form of free gas bubbles surrounded by a fluid or encapsulated bubbles used currently as contrast agents for medical echography, exhibit complex dynamics under specific acoustic excitations. Nonetheless, considering their micron size and the complexity of their interaction phenomenon with ultrasound waves, expensive and complex experiments and/or simulations are required for their analysis. The behavior of a microbubble along its equator can be linked to a system of coupled oscillators. In this study, the oscillatory behavior of a microbubble has been investigated through an acousto-mechanical analogy based on a ring-shaped chain of coupled pendula. Observation of parametric vibration modes of the pendula ring excited at frequencies between 1 and $5 \mathrm{~Hz}$ is presented. Simulations have been carried out and show mode mixing phenomena. The relevance of the analogy between a microbubble and the macroscopic acousto-mechanical setup is discussed and suggested as an alternative way to investigate the complexity of microbubble dynamics. (C) 2015 Acoustical Society of America. [http://dx.doi.org/10.1121/1.4936949]
\end{abstract}

Pages: $3600-3606$

\section{INTRODUCTION}

When subjected to an external acoustic field, bubbles can undergo complex radial oscillations. This oscillatory behavior has been an important and continuously developing research subject since the beginning of the 20th century. The investigation of bubble dynamics started with the work of Lord Rayleigh, ${ }^{1}$ who was mandated by the Royal Navy to explain the origin of damages on submarine propellers. Rayleigh focused on the oscillatory behavior of cavitation bubbles suspended in a fluid. He showed that the overpressure generated by the oscillations and the collapse of bubbles could explain the damages caused on propellers. In the 1930s, Minnaert was interested in the origin of the sound of running water. ${ }^{2} \mathrm{He}$ supposed that bubbles oscillating periodically in water were at the origin of rivers whispering. After these seminal works, many studies on bubble oscillations have been carried out in different research fields.

In addition to the radial motion, bubbles can show nonspherical oscillations or vibration modes. These vibration modes, characterized by an index $n$, were first analyzed theoretically at the interface between immiscible and incompressible fluids with spherical symmetries. ${ }^{3}$ Later, Neppiras ${ }^{4}$ analyzed the acoustic response from gas bubbles suspended in a fluid and subjected to sound fields. Eller and $\mathrm{Crum}^{5}$ and Prosperetti et al. ${ }^{6}$ focused on the instability of the motion and the nonlinear dynamics of a bubble within a sound field. In the 1990s, the discovery of single bubble sonoluminescence (SBSL) by Gaitan et al..$^{7}$ led to additional studies on nonlinear oscillations of bubbles. ${ }^{8-10}$ Finally, with the use of

a)Electronic mail: chalinejennifer@gmail.com ultrasound contrast agents, ${ }^{11}$ the understanding of bubble dynamics have found a renewed interest in the field of ultrasound imaging and targeted drug delivery. ${ }^{12,13}$ Therefore, the problem of vibration modes in bubbles is still under investigation.

In this study, the interaction between ultrasound and a microbubble, and especially the appearance of vibration modes, are studied using a macroscopic analog system. ${ }^{14,15}$ The mechanisms underlying its nonlinear behavior are sought to improve our understanding of the microbubble dynamics. The study of a single microbubble is a difficult task, particularly because of the interaction with other microbubbles or microstreaming that sweep away the bubble. Moreover, the smallness and the complexity of the phenomena involved, require complex modeling and expensive experiments. In this work, we propose the use of a macroscopic mechanical analog as an alternative way to investigate microbubble dynamics.

The study of analog models in the field of physics is a tool that allows recreating in the laboratory phenomena that are difficult to observe directly. Concerning microbubbles, some of its dynamic features (vibration modes, subharmonic oscillations, chaos) can be captured by a system of coupled oscillators. Here, the oscillatory behavior of a microbubble and its vibration modes are investigated through the use of a macroscopic analogy consisting of a chain of coupled pendula, parametrically excited by a vertical force. Based on a discrete nonlinear model of coupled pendula and its continuous limit describing low frequency excitations, vibration modes are investigated theoretically and experimentally. This approach is used here to set up a formal basis for the acousto-mechanical analogy of a gas microbubble. 
The structure of the paper is as follows: Mathematical formulations of a gas microbubble in an ultrasound field, and the analog acousto-mechanical system are given and compared in Secs. II and III, respectively. The experimental setup and the results of the measurement of vibration modes in the macroscopic system are described in Sec. IV, where numerical simulations are also presented. The conclusions of the study are presented in Sec. V.

\section{MICROBUBBLE DYNAMICS}

In the presence of an acoustic field, microbubbles can be forced to oscillate in different ways. The most common oscillation mode is the radial mode (Fig. 1) with the index $n=0$, and where the bubble compresses and expands radially, maintaining its spherical shape. The basic model describing the radial dynamics of a bubble is the Rayleigh-Plesset equation for the time-dependent radius $R$,

$$
\rho\left(R \ddot{R}+\frac{3}{2} \dot{R}^{2}\right)=P_{g}-P_{0}-P_{A}(t)-\frac{2 \sigma}{R}-4 \mu \frac{\dot{R}}{R},
$$

with $\rho$ the density of the surrounding fluid, $P_{g}=\left[P_{0}\right.$ $\left.+\left(2 \sigma / R_{0}\right)\right]\left(R_{0} / R\right)^{3 \gamma}$, the gas pressure inside the bubble, $P_{0}$ is the hydrostatic pressure, $R_{0}$ is the equilibrium radius of the bubble, $P_{A}(t)$ the acoustic pressure, $\sigma$ the surface tension of the bubble, $\mu$ the dynamic viscosity and $\gamma$ the polytropic exponent. Some generalizations of this model have been proposed. ${ }^{16-19}$ The resonance frequency of the radial mode of the bubble, the so called Minnaert frequency, can be obtained from linearization of Eq. (1) and is given by

$$
f=\frac{1}{2 \pi R_{0}}\left(\frac{3 \gamma P_{0}}{\rho}\right)^{1 / 2} .
$$

Under typical conditions, the relation $f \cdot R_{0}$ is a magnitude of order 1 . In particular, for a bubble in water at standard pressure $\left(P_{0}=100 \mathrm{kPa}, \rho=1000 \mathrm{~kg} / \mathrm{m}^{3}, \gamma=1.4\right)$, this equation gives the condition $f \cdot R_{0} \approx 3 \mathrm{~m} / \mathrm{s}$.

Microbubbles can also undergo non-spherical oscillations (see Fig. 2, left column) through instabilities at the
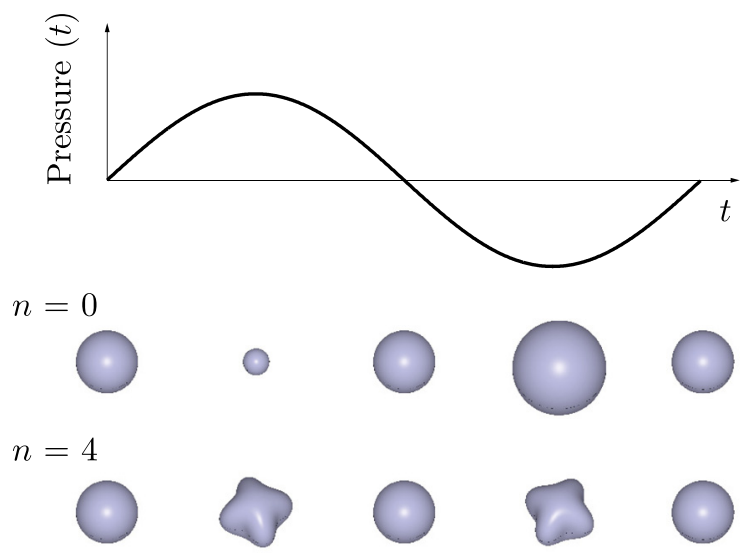

FIG. 1. (Color online) A bubble oscillating volumetrically within an acoustic field. For positive and negative pressures, compression and expansion phases are observed, respectively. Several modes can be observed: the radial mode $n=0$ and the non-spherical mode $n=4$. gas/fluid interface. In this case, the radius becomes a spacedependent function,

$$
R(t) \rightarrow R(\theta, \varphi, t)=R(t)+\xi(\theta, \varphi, t)
$$

where $R(t)$ describes the evolution of the radial mode and $\xi(\theta, \varphi, t)$ a perturbation depending on the spherical coordinates $\theta$ and $\varphi$. As Fig. 2 (left) shows, microbubbles can develop different surface patterns that depend on the excitation parameters (amplitude and frequency of the ultrasound wave) and the bubble radius. The radial oscillation corresponds to the mode $n=0$; the mode $n=1$ corresponds to the displacement of the center of mass. Non-spherical surface modes are those modes with $n \geq 2$.

To describe non-spherical modes in bubbles, a common analytical approach is to expand the perturbation of the radial mode, $\xi(\theta, \varphi, t)$, on the basis of spherical harmonics $^{3}$

$$
\xi(\theta, \varphi, t)=\sum_{n, m} a_{n}(t) Y_{n}^{m}(\theta, \varphi),
$$

where $a_{n}(t)$ is the time-dependent amplitude of the surface mode of index $n$, and $Y_{n}^{m}(\theta, \varphi)$ is the spherical harmonic defined as

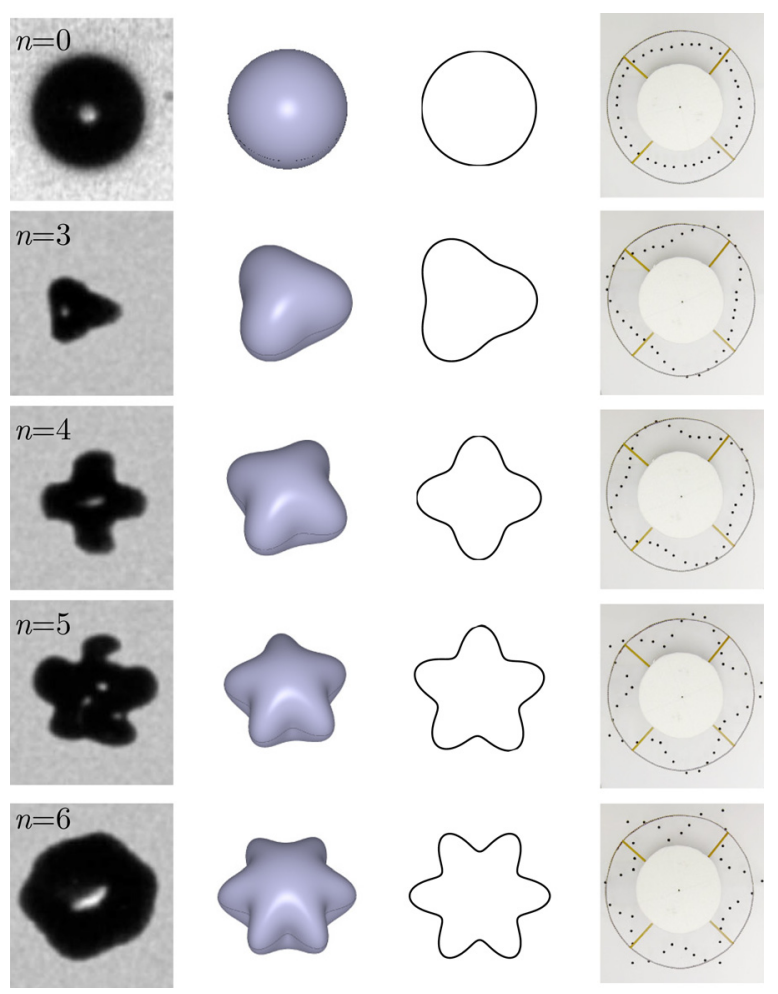

FIG. 2. (Color online) Representation of microbubble surface modes with $n$ the mode's order. The left pictures are experimental results from Ref. 20, and show a selection of surfaces modes observed for different microbubble radius between $36-45 \mu \mathrm{m}$. The two columns in the center are $3 \mathrm{D}$ analytical solutions from Eq. (5), and its equatorial cross section. The right column shows the corresponding vibration modes experimentally observed with the acousto-mechanical system, as described in Sec. IV. 


$$
Y_{n}^{m}(\theta, \varphi)=\frac{(-1)^{m}}{\sqrt{4 \pi}} \sqrt{\frac{(n-m) !}{(n+m) !}} \sqrt{2 n+1} P_{n}^{m}(\cos \theta) e^{i m \varphi},
$$

where $P_{n}^{m}$ are Legendre polynomials. Although $Y_{n}^{m}(\theta, \varphi)$ defines a large set of possible surface modal oscillations, experiments $^{20}$ show that the observed modes present symmetry along the axis of the incident ultrasound beam, corresponding to $m=0$, also known as zonal harmonics. Then, spherical harmonics $Y_{n}^{0}(\theta, \phi)$ are proportional to Legendre polynomials $P_{n}(\cos \theta)$. The evolution equation for the amplitude of each mode can be found by matching velocity potentials and pressures at both sides of the interface, and linearizing for small amplitudes, ${ }^{3}$

$$
\begin{aligned}
\ddot{a}_{n} & +\left(\frac{3 \dot{R}}{R}+\frac{2(n+2)(2 n+1)}{\rho R^{2}} \mu\right) \dot{a}_{n} \\
& +\left(\frac{(n+1)(n+2) \sigma}{\rho R^{3}}+\frac{2(n+2) \mu \dot{R}}{\rho R^{3}}-\frac{\ddot{R}}{R}\right) \\
& \times(n-1) a_{n}=0,
\end{aligned}
$$

where, $\mu$ is the viscosity and $\sigma$ the surface tension. Defining $b(t)=a(t) R^{3 / 2}$, the equation can be simplified as ${ }^{21}$

$$
\ddot{b}_{n}+\left(\frac{(n-1)(n+1)(n+2) \sigma}{\rho R^{3}}-\frac{3 \dot{R}^{2}}{4 R^{2}}-\frac{(2 n+1) \ddot{R}}{2 R}\right) b_{n}=0 .
$$

Within this approach, each mode $n$ obeys to the equation of a harmonic oscillator, with time-dependent coefficients. The resonance frequencies of the surface modes readily follow from Eq. (7) by considering the static condition $R=R_{0}$, and $\dot{R}=\ddot{R}=0$,

$$
\omega_{n}=\sqrt{\frac{(n-1)(n+1)(n+2) \sigma}{\rho R_{0}^{3}}},
$$

which is the Lamb expression for surface modes for a free gas bubble.

The acoustic pressure term $P_{A}(t)$ in Eq. (1) is usually a harmonic function with angular frequency $\omega_{e}$, i.e., $P_{A}(t)$ $=p_{A} \cos \left(\omega_{e} t\right)$. For sufficiently small amplitudes $p_{A}$, the bubble response will be also harmonic at the same frequency, i.e.,

$$
R(t)=R_{0}+R_{\varepsilon} \cos \left(\omega_{e} t\right),
$$

with $R_{\varepsilon} \ll R_{0}$. Substituting Eq. (9) into Eq. (7) and linearizing, a Mathieu equation for each surface mode is obtained: ${ }^{21}$

$$
\ddot{b}_{n}+\left[\omega_{n}^{2}+\left(\frac{(2 n+1) \omega_{e}^{2}}{2}-3 \omega_{n}^{2}\right) \frac{R_{\varepsilon}}{R_{0}} \cos \left(\omega_{e} t\right)\right] b_{n}=0
$$

The Mathieu equation is a special case of a linear second-order homogeneous differential equation with time-dependent coefficients, and appears in many applications in physics and engineering, specially in the description of parametrically driven systems, ${ }^{22}$ as the systems considered in this work.

\section{THE PARAMETRICALLY DRIVEN CHAIN OF COUPLED PENDULA}

In this section the model equations for the macroscopic analog of the microbubble are formulated. First, we consider the exact problem of the discrete lattice of coupled masses, which corresponds to our experimental system. Later, the continuum limit of this model is used to establish the analogy with gas microbubble, by deriving an equation isomorphic to Eq. (10).

\section{A. The discrete lattice}

The equation of motion of a pendulum of length $L$ is given by

$$
\ddot{\Theta}+\omega_{0}^{2} \sin \Theta=0,
$$

where $\Theta(t)$ is the angle with respect to the vertical, and $\omega_{0}=\sqrt{g / L}, g$ being the acceleration due to gravity. When a set of oscillators are coupled to their nearest neighbors, they form a lattice or chain, supporting waves. Consider that the lattice is subjected to a parametric forcing with displacement amplitude $h_{e}$ and angular frequency $\omega_{e}$, then its motion is described by

$$
\ddot{\Theta}_{i}+\left(\omega_{0}^{2}+\eta \cos \omega_{e} t\right) \sin \Theta_{i}-c^{2}\left(\Theta_{i+1}-2 \Theta_{i}+\Theta_{i-1}\right)=0,
$$

where $\Theta_{i}$ is the angle of the $i$ th pendulum, $\eta=4 \omega_{e}^{2} h_{e} / L$ is the forcing parameter, and $c$ is a constant denoting the strength of the coupling, equivalent to the speed of sound for the waves for the lattice waves. Lattice models like Eq. (12) have been extensively studied, in the context of FrenkelKontorova chain. ${ }^{23}$ Most of the work is theoretical, and focused on the formation of localized states (breathers, solitons, and kinks). There are few experimental works, ${ }^{24-26}$ where different parametric driving mechanisms and coupling have been implemented. In Ref. 24, coupling is achieved by strings and knots, while in Ref. 26 torsional springs are used. In all the cases, a linear arrangement of pendula was considered. Boundary conditions typical of this configuration are either fixed or free ends. For the sake of simplicity, in the forthcoming analytical treatment we have ignored dissipation, which is small but finite in our experiment. It can be added as a phenomenological term in Eq. (12) by including an additional term $\beta \dot{\Theta}$. Our numerical simulations later will take into account such dissipative term, with $\beta=5 \times 10^{-4}$.

Here, in order to mimic a bubble-like behavior, we consider the chain in a circular arrangement. This implies the periodic boundary condition $\Theta_{N+1}=\Theta_{1}$. In addition to this, the circular configuration incorporates curvature effects, which are not present in the linear chain. We assume a V-shaped coupling [see Fig. 3(a)], for which coupling strength is given by 
a)

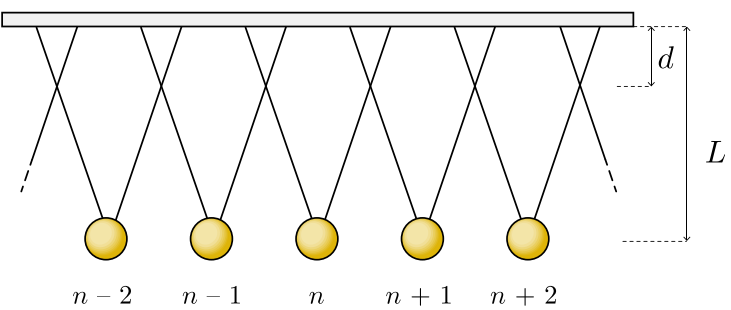

b)

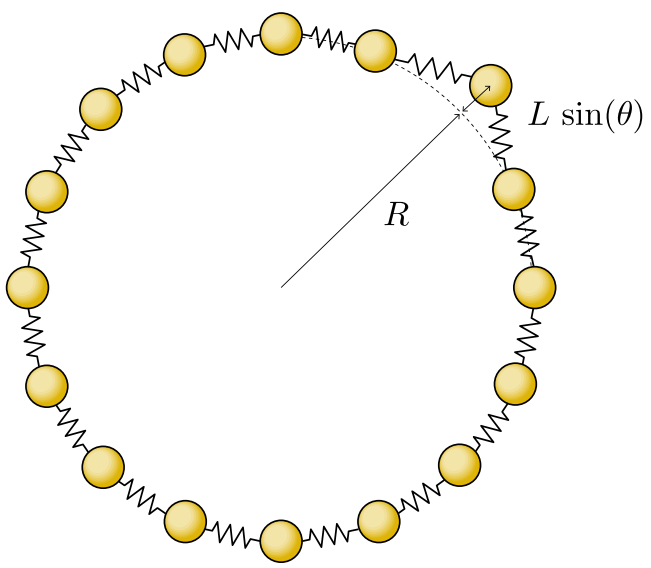

FIG. 3. (Color online) Schematic representation of the chain of pendula coupled by $\mathrm{V}$-shaped strings and knots, hanging from a rigid support. (a) Lateral view of a section of the chain, (b) top view of the whole chain in a circular arrangement. Deviation of one mass with respect to its equilibrium position, and its coordinates are shown for illustration.

$$
c^{2}=\frac{g d a^{2}}{4 L(L-d)},
$$

where $d$ is the distance from the knot to the support, and $a$ the distance between masses. ${ }^{24}$ Note that, in this model, the coupling strength can be varied by selecting the position of the knot.

The chain supports different oscillation modes, labeled with an integer index $n$, corresponding to standing waves with wavenumber $k_{n}$. For the circular chain of radius $R_{0}$, the relation $k_{n}=n / R_{0}$ holds. The relation between driving frequency and mode index (the dispersion relation) can be obtained, for negligible damping and forcing, after linearization and assuming a solution in the form of a harmonic wave, $\exp i(k n a-\omega t)$. This results in the relation

$$
\omega_{n}=\sqrt{\frac{g}{L-d}\left[1-\frac{d}{L} \cos ^{2}\left(\frac{n a}{2 R_{0}}\right)\right]} .
$$

As shown in Fig. 3(b), which illustrates the chain viewed from the top, the ring of oscillators is equivalent to an equatorial section of a bubble with equilibrium radius $R_{0}$,

$$
R_{i}(t)=R_{0}+L \sin \Theta_{i}(t),
$$

where $R_{0}$ is the radius of the ring (equivalent to the equilibrium radius of the bubble). Assuming small angles $\sin \Theta_{i} \simeq \Theta_{i}$, the position of each pendulum at a time $t$ can be given by $R_{i}(t)=R_{0}+L \Theta_{i}(t)$.

\section{B. Continuous description}

The bubble surface is a continuum, while the chain of pendula is a discrete system. Therefore, analogies must be searched in the limit where a continuum version of Eq. (12) applies. This is the case when we restrict our analysis to long-wavelength modes (low index $n$ ), where the mode scale are much larger than the distance between two pendula, $k_{n} a \ll 1$, or $n a / R_{0} \ll 1$. Considering this limit, the discrete angle coordinates $\Theta_{i}(t)$ can be replaced by the continuous function $\Theta(x, t)$.

The equation of motion Eq. (12) leads to the parametrically driven, small amplitude, sine-Gordon model that reads

$$
\ddot{\Theta}-c^{2} \Theta_{x x}+\left[\omega_{0}^{2}+\eta \cos \left(\omega_{e} t\right)\right] \Theta=0,
$$

where $\Theta$ is a surface deformation related to the bubble radius as $\Theta=\left(R-R_{0}\right) / L$.

Solutions of Eq. (16) can be expressed as a superposition of normal modes. For a linear (straight) chain, a proper basis is given by the harmonic functions

$$
\Theta(x, t)=\sum_{n} b_{n}(t) \cos \left(k_{n} x\right) .
$$

Some solutions of Eq. (16) have been discussed for a onedimensional straight geometry. ${ }^{27,28}$

In the ring geometry considered here, angular coordinates are more suitable to describe the position of a point on the deformed ring, and we use the transformation $x=R \theta$, where $\theta=[0,2 \pi]$ and $R=R_{0}$ is the equilibrium radius. The Laplacian operator takes the form $\Theta_{x x}=R^{-2} \Theta_{\theta \theta}$. A proper basis for the expansion in this case is

$$
\Theta(\theta, t)=\sum_{n} b_{n}(t) P_{n}(\cos \theta),
$$

where $P_{n}(\cos \theta)$ is a Legendre polynomial and $\theta$ is the angular coordinate.

The equation for the temporal evolution of the mode amplitudes can be obtained by substituting Eq. (17) or Eq. (18) into Eq. (16) and projecting over the different modes (Galerkin projection) using the orthogonality properties of harmonic functions or Legendre polynomials. Independently of the basis chosen for the expansion, the following equation is obtained:

$$
\ddot{b}_{n}+\left[\omega_{n}^{2}+\eta \cos \left(\omega_{e} t\right)\right] b_{n}=0,
$$

which is a Mathieu equation, where the parametric excitation amplitude is $\eta=\left(4 \omega_{e}^{2} h_{e}\right) / L$ and the frequency on the $n$th mode is given by

$$
\omega_{n}^{2}=\omega_{0}^{2}+c^{2} \frac{n^{2}}{R_{0}^{2}} .
$$

The same result is obtained from the exact dispersion relation Eq. (14) for the discrete system, evaluated in the limit of low index $n$, where $n a / R_{0} \ll 1$. 


\section{Analogy}

In this paper, the analogy between the ultrasound driven gas bubble and the ring of coupled pendula subjected to a time-dependent acceleration is considered at different levels. One first level is just descriptive, where the mechanical analogue behaves similarly to the microbubble surface under an ultrasound field. The vertical forcing is equivalent to the ultrasound pressure field, in the sense that it excites parametrically the motion, and the chain of pendula play the role of the surface of the bubble, coupling represents surface tension, and so on. In a second level of analogy, we have shown that in both cases the product $f_{e} \cdot R_{0}$ can be chosen to be of the same order (the product is fixed for a gas microbubble, given by the Minnaert formula, however in the mechanical analogue the radius of the bubble can be chosen independently of the resonant frequency, which depends on the length of the pendula). So at this level the analogy become quantitative. The ring of pendula in this work, with $R_{0} \simeq 0.3 \mathrm{~m}$, was designed to be excited in the $\mathrm{Hz}$ frequency range. The third, and deeper level of analogy is based on the demonstration that the dynamics of both systems is described by the same Mathieu equation, whose coefficients depend on the specific system and can be obtained analytically in simple form. Such coefficients are listed in Table I, which is the main result of this work. Once the connections between the parameters in both systems are established, experimental investigation with the macroscopic analogue may result in insight of new phenomena in acoustically driven bubbles.

\section{EXPERIMENTS}

\section{A. Experimental setup}

The setup consists of an aluminum ring, on which pendula of mass $m=6 \mathrm{~g}$ are fixed with nylon strings forming a "V" shape with the vertical axis as shown in Fig. 3(a). The coupling between pendula is obtained by overlapping the strings and fixing them by a knot. ${ }^{24}$ The parameter with a stronger influence on the dynamics of the chain is the coupling strength. Here, we focus on a ring with a medium coupling. The ring has a radius $R=31 \mathrm{~cm}, N=54$ pendula, pendulum's length is $L=10 \mathrm{~cm}$ and the distance between the ring and the node is $d=5 \mathrm{~cm}$. As Fig. 4 shows, the pendula ring lies on the excitation system. The mechanical excitation system consists of a subwoofer loudspeaker driven by an arbitrary waveform generator (Agilent33220A, Agilent Technologies, Loveland, CO) through an audio

TABLE I. Table of analogies between parameters of microbubble and those of the pendula ring.

\begin{tabular}{lcc}
\hline \hline Parameter & Microbubble & Pendula ring \\
\hline$R_{0}[\mathrm{~m}]$ & $\simeq 10^{-6}$ & $\simeq 0.3$ \\
$f_{e}\left[\mathrm{~s}^{-1}\right]$ & $\simeq 10^{6}$ & $\simeq 3$ \\
$f_{e} \cdot R_{0}\left[\mathrm{~m} \mathrm{~s}^{-1}\right]$ & $\mathcal{O}(1)$ & $\mathcal{O}(1)$ \\
$\omega_{n}^{2}\left[\mathrm{~s}^{-2}\right]$ & $(n-1)(n+1)(n+2) \frac{\sigma}{\rho R_{0}^{3}}$ & $\frac{g}{L}+n^{2} \frac{c}{R_{0}^{2}}$ \\
$\eta\left[\mathrm{s}^{-2}\right]$ & $\frac{(2 n+1)}{2} \omega_{e}^{2}\left(\frac{R_{\varepsilon}}{R_{0}}\right)$ & $4 \omega_{e}^{2}\left(\frac{h_{e}}{L}\right)$ \\
\hline \hline
\end{tabular}

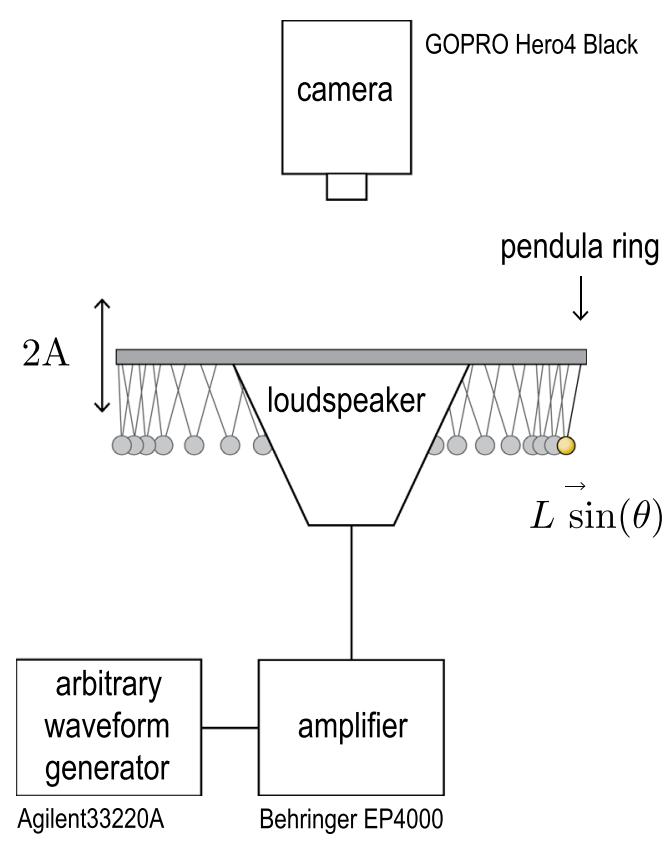

FIG. 4. (Color online) Scheme of the experimental setup. The ring of coupled pendula is driven vertically by an sinusoidal force generated by a loudspeaker.

amplifier. Thus, the pendula ring is attached to the subwoofer cone and therefore is excited mechanically by a vertical oscillatory force, as shown in Fig. 4 . The sinusoidal excitation varies from $f_{e}=0$ to $5 \mathrm{~Hz}$ with amplitudes varying from $A=0.5$ to $3.5 \mathrm{~V}_{\mathrm{pp}}$, corresponding to a vertical displacements ranging from $h_{e}=0.5$ to $3.5 \mathrm{~mm}$. The pendula are driven near the volumetric resonant frequency and its double to allow the development of surface modes during the mechanical excitation through parametric instability.

The motion of pendula has been recorded from the top with a video camera. The data processing have been performed with the software Image J and the plug-in MJ Track. This plug-in enables us to track the motion of each pendulum and thus to determine the distance $R_{i}(t)$ between the center of the ring and a pendulum at a fixed time $t$. Applying a spatial fast Fourier transform on $R_{i}(t)$, the amplitude of each vibration mode of order $n$ can be obtained.

\section{B. Results}

A set of measurements has been carried out for excitation amplitudes between $h_{e}=0.5$ and $3.5 \mathrm{~mm}$ and frequencies varying from $f_{e}=0$ to $5 \mathrm{~Hz}$. Vibration modes have been observed up to $n=22$, including an unstable volumetric mode for $n=0$. The mode $n=1$ corresponding to the displacement of the center of mass has also been observed. An example of the observed patterns is given in Fig. 5 for an excitation amplitude $h_{e}=2 \mathrm{~mm}$ and frequency $f_{e}=3.20 \mathrm{~Hz}$. Here, the pendula ring shows a mode $n=3$. A fit to the corresponding Legendre polynomial, shown in continuous line, shows a good agreement.

To compare with the experimental results, Eq. (12) has been integrated numerically by an explicit central finite differences scheme, with a temporal step $\Delta t=\left(2 \pi / \omega_{0}\right) 1000$. A set of solutions were simulated for amplitudes ranging from $h_{e}=0$ to $3.5 \mathrm{~mm}$ and excitation frequency ranging from 

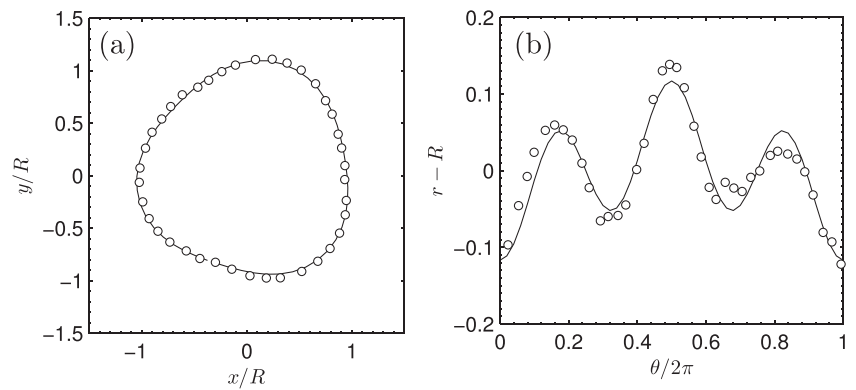

FIG. 5. Experimental mode $n=3$ (symbols) and the corresponding fit to a Legendre polynomial $P_{3}(\cos \theta)$. Two different representations, in Cartesian coordinates (a) and in polar coordinates (b).

$\omega_{e}=0.5$ to $3 \omega_{0} \mathrm{rad} / \mathrm{s}$. The amplitudes of the vibration modes were evaluated by a spatial fast Fourier transform of each numerical solution. A phase diagram showing the domain of existence of vibration modes $n$ for different excitation parameters is presented in Fig. 6. The numerical simulation of Eq. (12) evidences the existence of different sets of resonances. Due to the damping, there exists a threshold for the excitation of the different modes. The lowest threshold is obtained for the so-called $p / q=2: 1,(p, q$ integers) or subharmonic resonance, where the frequency of the excitation is twice the natural frequency of the pendulum. Within each resonance set, each mode has its own instability

(a)

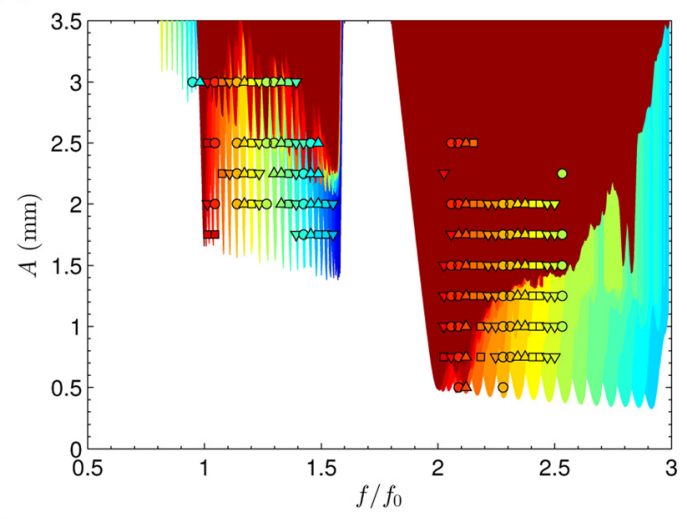

(b)

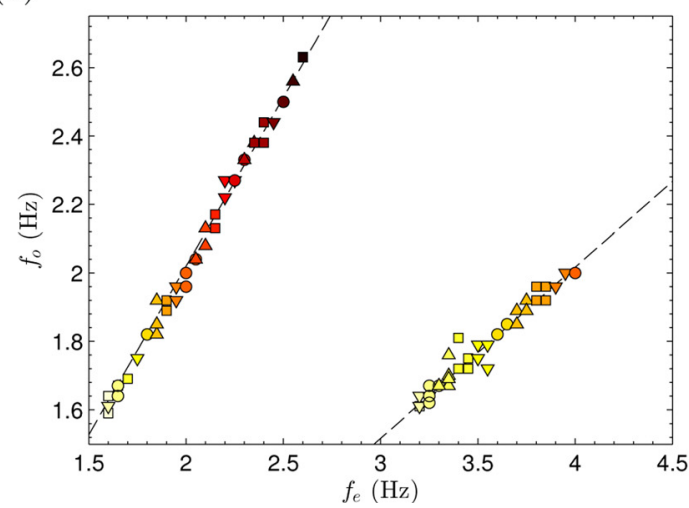

FIG. 6. (Color online) (a) Phase diagram showing the appearance of vibration modes for different excitation parameters. The symbols correspond to the experimental data whereas the colored areas are the instability regions obtained numerically. (b) Oscillation frequencies of the pendulum (denoted by $f_{0}$ ) versus the excitation frequency $\left(f_{e}\right)$. On both graphics, each symbol corresponds to a vibration mode. region, or Arnold tongues, which are represented in Fig. 6 with a different color for each mode. The dark shaded region corresponds to numerically unstable solutions. Note also the existence of a second set at lower frequencies, with a higher threshold, denoting the 1:1 resonance, which corresponds to modes excited when driving the system at the natural frequency of the pendulum $f_{0}=\sqrt{(g / L)} \approx 1.6 \mathrm{~Hz}$. The symbols in Fig. 6 correspond to experimental data. From left to right in each set, the mode number increases, similarly to the numerical results. No vibration modes are observed at frequencies below $f_{e} / f_{0}=0.95$, as there are no instability regions predicted by the theory. From $f_{e} / f_{0}=0.95$ to 1.90 , a first group of vibration modes appears when the excitation amplitude is greater than a threshold. These modes oscillate at a frequency equal to the excitation frequency $\left(f_{\text {osc }}=f_{e}\right)$, and correspond to the $p / q=1: 1$ parametric resonance of the system. From $f_{e} / f_{0}=1.90$ to $f_{e} / f_{0}=3.17$, a second group of vibration modes oscillating at subharmonics of the excitation frequency $\left(2 f_{\text {osc }}=f_{e}\right.$ ) is observed, corresponding to the 2:1 resonance. Therefore, two sets of resonance modes have been observed. Comparing the trend of simulation and experimental data shown in Fig. 6, we can conclude that there exists a good agreement.

It is important to note that pure modes exist only in a narrow region. Close to the parametric instability threshold, the Arnold tongues corresponding to each mode $n$ overlap. Thus, most of the modes observed actually correspond to a mixing of neighboring modes, with one mode being clearly dominant. Mode mixing is therefore expected to occur also in ultrasound-driven bubbles; however, such mode mixing has been postulated but not analyzed or observed in such microbubbles. The mechanical analog presented here could be used to explore these complex phenomena.

Finally, we note that the maximum excitation has been measured at $f_{e}=5 \mathrm{~Hz}\left(f_{e} / f_{0}=3.2\right)$. Above this frequency, measurements cannot be performed due to the setup vulnerability. Oscillations becomes jerky and the coupling between pendula often breaks.

\section{CONCLUSIONS}

The interaction between a microbubble and an acoustical field has been studied through the use of a macroscopic acousto-mechanical analogy. As for real bubbles, vibration modes and modes mixing have been observed. It is known that, in the case of microbubbles, vibration modes display a strong subharmonic behavior. Here, with the pendula ring, vibration modes have also been excited in the region where $f_{\text {osc }}=f_{e}$, corresponding to a $p / q=1: 1$ resonance, $p, q$ integer.

Localized modes like breather have not been discussed here but a previous study on the pendula ring shows the appearance of such modes. ${ }^{29,30}$ One can thus expect to observe these oscillatory behaviors in microbubbles. These results allow us to offer new insights in the study of microbubble's dynamics. The analogy between the macroscopic behavior of the pendula ring and the microscopic behavior of a microbubble presented here is valid for low order modes (i.e., in the limit $n a / R_{0} \ll 1$ ) and provides important insights 
for the comprehension of microbubble dynamics. Further studies are underway, particularly an expansion of the analogy to consider encapsulated microbubbles to get information for both imaging and therapeutic applications using contrast microbubbles.

\section{ACKNOWLEDGMENTS}

Thanks to Jean-Marc Grégoire and Jean-Yves Tartu for their fruitful help in the design of the experimental setup. J.C. was the recipient of a Ph.D. fellowship from the Région Centre Val de Loire (France). Financial support from the University Francois-Rabelais doctoral school of Tours was granted to finance a research stay on this topic at the Universidad Politecnica de Valencia (Spain). A.M. is recipient of a research fellowship from Generalitat Valenciana (Santiago Grisolia program).

${ }^{1}$ Lord Rayleigh, "On the pressure developed in a liquid during the collapse of a spherical cavity,” Philos. Mag. 34, 94-98 (1917).

${ }^{2}$ M. Minnaert, "On musical air-bubbles and the sounds of running water," Philos. Mag. Ser. 7 16(104), 235-248 (1933).

${ }^{3}$ M. S. Plesset, "On the stability of fluid flows with spherical symmetry," J. Appl. Phys. 25(1), 96-98 (1954).

${ }^{4}$ E. A. Neppiras, "Subharmonic and other low-frequency emission from bubbles in sound-irradiated liquids," J. Acoust. Soc. Am. 46(3B), 587-601 (1969).

${ }^{5}$ A. I. Eller and L. A. Crum, "Instability of the motion of a pulsating bubble in a sound field," J. Acoust. Soc. Am. 47(3B), 762-767 (1970).

${ }^{6}$ A. Prosperetti, L. A. Crum, and K. W. Commander, "Nonlinear bubble dynamics," J. Acoust. Soc. Am. 83(2), 502-514 (1988).

${ }^{7}$ D. F. Gaitan, L. A. Crum, C. C. Church, and R. A. Roy, "Sonoluminescence and bubble dynamics for a single, stable, cavitation bubble," J. Acoust. Soc. Am. 91, 3166-3183 (1992).

${ }^{8}$ M. P. Brenner, D. Lohse, and T. F. Dupont, "Bubble shape oscillations and the onset of sonoluminescence," Phys. Rev. Lett. 75, 954-957 (1995).

${ }^{9}$ F. Gaitan and G. Holt, "Nonlinear bubble dynamics and light emission in single-bubble sonoluminescence," J. Acoust. Soc. Am. 103, 3046 (1998).

${ }^{10}$ U. H. Augsdörfer, A. K. Evans, and D. P. Oxley, "Thermal noise and the stability of single sonoluminescing bubbles," Phys. Rev. E 61, 5278-5286 (2000).

${ }^{11}$ J. Ophir and K. J. Parker, "Contrast agents in diagnostic ultrasound," Ultrasound Med. Biol. 15(4), 319-333 (1989).

${ }^{12}$ J.-M. Escoffre, A. Novell, A. Zeghimi, and A. Bouakaz, "Ultrasound and microbubbles for in vitro gene delivery," Drug Dev. Delivery 11(2), 42-47 (2011)

${ }^{13}$ A. Novell, J.-M. Escoffre, and A. Bouakaz, "Ultrasound contrast imaging in cancer technical aspects and prospects," Curr. Mol. Imaging 2(1), $77-88$ (2013).
${ }^{14}$ S. Dos Santos, V. Sanchez-Morcillo, N. Jimenez, J. Chaline, and A. Bouakaz, "Modulational instability and localized modes for ultrasound contrast microbubbles surface oscillations," in 2011 IEEE International Ultrasonics Symposium (IUS), (2011), pp. 648-651.

${ }^{15}$ S. Dos Santos, V. Sánchez-Morcillo, N. Jiménez, A.-P. Abellard, and A. Bouakaz. "Modulational instability of microbubbles surface modes," AIP Conf. Proc. 1433(1), 311-314 (2012).

${ }^{16} \mathrm{C}$. C. Church, "The effects of an elastic solid surface layer on the radial pulsations of gas bubbles," J. Acoust. Soc. Am. 97(3), 1510-1521 (1995).

${ }^{17}$ L. Hoff, P. C. Sontum, and J. M. Hovem, "Oscillations of polymeric microbubbles: Effect of the encapsulating shell," J. Acoust. Soc. Am. 107(4), 2272-2280 (2000).

${ }^{18} \mathrm{D}$. Chatterjee and K. Sarkar, "A Newtonian rheological model for the interface of microbubble contrast agents," Ultrasound Med. Biol. 29(12), 1749-1757 (2003).

${ }^{19}$ P. Marmottant, S. van der Meer, M. Emmer, M. Versluis, N. de Jong, S. Hilgenfeldt, and D. Lohse, "A model for large amplitude oscillations of coated bubbles accounting for buckling and rupture," J. Acoust. Soc. Am. 118(6), 3499-3505 (2005).

${ }^{20}$ M. Versluis, D. E. Goertz, P. Palanchon, I. L. Heitman, S. M. van der Meer, B. Dollet, N. de Jong, and D. Lohse, "Microbubble shape oscillations excited through ultrasonic parametric driving," Phys. Rev. E 82, 026321 (2010).

${ }^{21}$ T. G. Leighton, The Acoustic Bubble (Academic Press, Harcourt Brace \& Company, London, 1994), Chap. 4.

${ }^{22}$ L. Ruby, "Applications of the Mathieu equation," Am. J. Phys. 64, 39-44 (1996).

${ }^{23}$ O. M. Braun and Y. S. Kivshar, The Frenkel-Kontorova Model: Concepts, Methods and Applications (Springer-Verlag, Berlin, 2004), 472 pp.

${ }^{24}$ B. Denardo, B. Galvin, A. Greenfield, A. Larraza, S. Putterman, and W. Wright, "Observations of localized structures in nonlinear lattices: Domain walls and kinks," Phys. Rev. Lett. 68, 1730-1733 (1992).

${ }^{25}$ W.-Z. Chen, "Experimental observation of solitons in a 1D nonlinear lattice," Phys. Rev. B. 49, 15063-15066 (1994).

${ }^{26}$ B. Thakur, L. Q. English, and A. J. Sievers, "Driven intrinsic localized modes in a coupled pendulum array," J. Phys. D: Appl. Phys. 41, 015503 (2008).

${ }^{27}$ D. Armbruster, M. George, and I. Oprea, "Parametrically forced pattern formation," Chaos 11, 52-56 (2001).

${ }^{28}$ J. Cuevas, L. Q. English, P. G. Kevrekidis, and M. Anderson, "Discrete breathers in a forced-damped array of coupled pendula: Modeling, computation, and experiment," Phys. Rev. Lett. 102, 224101 (2009).

${ }^{29}$ V. Sanchez-Morcillo, N. Jimenez, S. Dos Santos, J. Chaline, A. Bouakaz, and N. Gonzalez, "Modeling acoustically driven microbubbles by macroscopic discrete-mechanical analogues," Modell. Sci. Educ. Learn. 6, 75-87 (2013).

${ }^{30}$ V. Sanchez-Morcillo, N. Jimenez, J. Chaline, A. Bouakaz, and S. Dos Santos, "Spatio-temporal dynamics in a ring of coupled pendula: Analogy with bubbles," in Localized Excitations in Nonlinear Complex Systems (Springer International, Switzerland, 2014), pp. 251-262. 